\title{
An Efficient Lattice-based Distributed IBE
}

\author{
Lu YIN, Quan ZHANG, and Rui-Lin LI \\ School of Electronic Science and Engineering, National University of Defense Technology, NUDT, Changsha, China \\ ruruyin@163.com,quanzhang@nudt.edu.cn, securityırl@163.com
}

\begin{abstract}
This paper improves the lattice-based secret sharing method presented by Bansarkhani and Meziani and combines the method with the IBE based on the standard LWE problem constructing an efficient lattice-based distributed decryption IBE. Our construction avoids the weakness in the traditional method like Lagrange interpolation, Blakley`s space geometry method or Chinese remainder theorem method. Through theoretical analysis, compared with the bilinear pairing based distributed IBE, our scheme refrains from the complex pairing operation and has less calculation cost. A proof of security holds in the standard model.
\end{abstract}

\section{Introduction}

In 1984, Shamir proposed the concept of Identity-Based Cryptography(IBC)[1]. The characteristic of IBC is that a user's public key can be calculated by an open algorithm according to some public information like the user's name, e-mail address or phone number, etc. The property simplifies public key distribution. In this aspect, the IBC has an advantage over traditional public key cryptosystem. Since the user's private key must be created by a trusted third party $\mathrm{PKG}$, it results in the inherent key escrow problem and restricts the adoption of IBC.

In 2003, Boneh and Franklin designed the first practical Identity-Based Encryption scheme (IBE)[2] using bilinear maps on elliptic curves and proposed an approach to mitigate the key escrow problem. In this approach, the IBE system's master key is distributed to multiple PKGs using technique of Shamir $(t, n)$ threshold secret sharing[3]. The BasicIdent scheme is briefly described below:

Let $G_{1}$ be an additive cyclic group of order $p$ and $G_{T}$ be a multiplicative group of order $\mathrm{p}, \mathrm{P}$ is a generator of $\mathrm{of}_{1}$, $\hat{e}: G_{1} \times G_{1} \rightarrow G_{T}$ is a bilinear map.

Let $\mathrm{H}_{1}$ and $\mathrm{H}_{2}$ be two cryptographic hashfunctions $H_{1}:\{0,1\}^{*} \rightarrow G_{1}, H_{2}: G_{T} \rightarrow\{0,1\}^{l m}$, where ${ }^{l_{m}}$ represents the bit length of the message.
1) Setup. The master PKG chooses a random master key $s \in \mathbf{Z}_{p}^{*}$ and sets $P_{p u b}=s P . Q_{i d}=H_{1}(i d)$ is derived from the user's public key id. The master PKG then distributes sto $\mathrm{nPKGs}$ in a Shamir $(\mathrm{t}, \mathrm{n})$ threshold secret sharing fashion, each $\mathrm{PKG}_{\mathrm{i}}$ gets one shares . $_{\text {. }}$

2) Encrypt. The sender picks a random $r \in \mathbf{Z}_{p}^{*}$ and encrypts the plaintext under the public key id, setting the ciphertext to be

$$
C=(U, V)=\left(r P, m \oplus H_{2}\left(\hat{e}\left(Q_{i d}, r P_{p u b}\right)\right)\right) .
$$

3) Decrypt. After receiving the ciphertext $C$, userid sends decryption request $R=(i d, U)$ to $\mathrm{PKG}_{\mathrm{i}}$. When responding to the request more than $\mathrm{t}$ ( $\mathrm{t}$ is the threshold value) $\mathrm{PKG}_{\mathrm{i}}$ send $\hat{e}\left(s_{i} Q_{i d}, U\right)$. Useridcan then construct plaintext $\mathrm{m}$ via $m=V \oplus H_{2}\left(\prod_{i=1}^{t} \hat{e}\left(\lambda_{i} s_{i} Q_{i d}, U\right)\right)=V \oplus H_{2}\left(\hat{e}\left(s Q_{i d}, U\right)\right)$ where the $\lambda_{\mathrm{i}}$ 's are the Lagrange coefficients.

In this scheme, each distributed PKG can either generate the decryption share $\hat{e}\left(s_{i} Q_{i d}, U\right)$ online or provide the partial private key $s Q_{i d}$ for the user. Of course, the complete private key can be obtained when the collusion of more than $\mathrm{t}$ PKGs happens. Thus the honesty of these entities required 
in the application is comparable to $\mathrm{PKG}$, which is the essential reason for these entities to be PKGs. Distributed PKGs required to be involved in the decryption online all the time, it violates the basic requirement of IBE, PKG can be offline after generating the private keys for the users in its domain, that Shamir proposed[1].

To solve this problem, in PKC 2004, Baek and Zheng Yuliang [4] gave a better approach to share a private key associated with an identity among $\mathrm{n}$ entities which appointed by user rather than sharing a master key. So these $n$ entities are downgraded to pure decryption servers without the function of PKG. Because these servers have users' trust and the online/offline state controlled by the user, the problem of distributed PKGs online all the time in the decryption period can be solved.

However, due to the distributed decryption IBE in [4] is based on bilinear pairing and Shamir threshold secret sharing, considering the complex computation of pairing operation, it affects the overall efficiency of the protocol. In addition, from the point of view of security, their scheme is hard to resist quantum attacks.

In recent years, constructing IBE from some hard problems in lattice attracts much attention. In 2008, under the random oracle model Gentry, Peikert and Vaikuntanathan[5] constructed the first IBE based on the LWE problem in the lattice. Subsequently, Cash, Hofheinz and Kiltz[6], Agarwal, Boneh and Boyen[7] proposed lattice based IBE in the standard model. Since the operation on the lattice is mostly linear and the calculation speed is fast, so we combine the idea of distributed decryption presented by Baek and Zheng Yuliang[3] with efficient lattice IBE[7] to construct an efficient lattice-based distributed IBE.

If our construction follows the Lagrange polynomial interpolation used in Baek and Zheng Yuliang's scheme to distribute a secret n-dimensional vector, the communication cost will increase because the order of each vector component has to be recorded to recover the n-dimensional vector. Therefore, our construction uses the secret sharing approach presented by R. Bansarkhani and M. Meziani[8] to distribute the user's private key which is an n-dimensional vector in the lattice based IBE. However we argue that there is a scalability problem in the secret sharing construction of R. Bansarkhani and M. Meziani. We propose to employ a variable number of columns of a matrix to distribute the n-dimensional vector so that the number of decryption servers is available within a certain range.

The advantages of our lattice based distributed IBE are listed as follows:

-In the distribution and decryption share construction/reconstruction stage, the new secret sharing technique based on the variable matrix mitigates the scalability problem and avoids the weakness of recording the order of each vector component while recovering the whole n-dimensional vector using traditional methods like Lagrange polynomial interpolation[2], Blakley's geometry of space scheme[9] or Chinese remainder theorem method[10], etc.
-The secret shares and the reconstructed private key can be verified by a lattice-based hash function.

-The user makes a decryption request to each decryption server after receiving the ciphertext, then the decryption servers calculate decryption shares associated with the ciphertext and secret shares they possess. Through a secure channel, the corresponding decryption shares are delivered to the user. The whole encryption and decryption process including secret shares distribution, the generation of decryption shares or recovering the plaintext uses simple matrix operations. Through the theoretical analysis, compared with the distributed bilinear pairing-based IBE, our construction can not only resist quantum attacks, and also reduce the cost of calculation.

\section{Preliminaries}

\subsection{Notation}

The set of real numbers is defined as $\mathbf{R}$ and the integers as $\mathbf{Z}$. We denote a vector $x$ and the $i_{\text {th }}$ component of $x$ is written as $x^{(i)}$.

Let $\mathrm{S}$ be a set of vectors $S=\left\{s_{1}, . ., s_{k}\right\}$, where $s_{1}, \ldots, s_{k} \in \mathbf{R}^{m}$. We define the $\mathrm{L}_{2}$ length of the longest vector in $\mathrm{S}$ as $\|S\|$, namely $\|S\|:=\max _{1 \leq i \leq k}\left\|s_{i}\right\|$. We let $\tilde{S}:=\left\{\tilde{S}_{1}, \ldots, \tilde{S}_{k}\right\}$ denote the Gram-Schmidt orthogonalization of the vectors $s_{1}, \ldots, s_{k}$ and $\|\tilde{S}\|$ denote the Gram-Schmidt norm of S.

The statistical distance between two distributions $\mathrm{X}$ and $\mathrm{Y}$ over a finite set $\Omega$ is defined as:

$$
\Delta(\mathrm{X} ; \mathrm{Y}):=\frac{1}{2} \sum_{s \in \Omega}|\operatorname{Pr}[\mathrm{X}=\mathrm{s}]-\operatorname{Pr}[\mathrm{Y}=\mathrm{s}]|
$$

Two distributions are statistically close if $\Delta(\mathrm{X} ; \mathrm{Y})$ is negligible. Let $U_{\Omega}$ be a uniform distribution over $\Omega$. If $\Delta\left(\mathrm{X} ; U_{\Omega}\right) \leq \delta$, we say $\mathrm{X}$ is $\delta$-uniform over $\Omega$.

\subsection{Integer Lattices}

An integer lattice is a discrete subgroup of $\mathbf{Z}^{m}$. Our article will use the q-ary integer lattices to construct the lattice-based IBE. The q-ary integer lattices contain $q \mathbf{Z}^{m}$ as a sublattice where $\mathrm{q}$ is some integer. Let $\mathrm{q}$ be a prime, $\mathrm{A} \in \mathbf{Z}_{q}^{n \times m}$ be arbitrary, $u \in \mathbf{Z}_{q}^{n}$, define the full-rank q-ary lattice:

$$
\Lambda_{q}^{\perp}(\mathrm{A}):=\left\{\mathrm{e} \in \mathbf{Z}^{m} \mid \mathrm{Ae}=0(\bmod \mathrm{q})\right\} .
$$

The coset or shifted lattice is defined to be: 


$$
\Lambda_{q}^{u}(\mathrm{~A}):=\left\{\mathrm{e} \in \mathbf{Z}^{m} \mid \mathrm{Ae}=u(\bmod \mathrm{q})\right\}
$$

For $t \in \Lambda_{q}^{u}(\mathrm{~A}), \Lambda_{q}^{u}(\mathrm{~A})=\Lambda_{q}^{\perp}(\mathrm{A})+t$.

\subsection{Discrete Gaussians}

For any $c \in \mathbf{R}^{m}$, real $\sigma>0$, the m-dimensional Gaussian function $\rho_{\sigma, c}(x)$ is defined by:

$$
\forall x \in \mathbf{R}^{m}, \rho_{\sigma, c}(x)=\exp \left(-\pi \frac{\|x-c\|^{2}}{\sigma^{2}}\right) .
$$

For any lattice $\Lambda \subset \mathbf{R}^{m}, \quad \rho_{\sigma, c}(\Lambda)=\sum_{x \in \Lambda} \rho_{\sigma, c}(\mathrm{x})$. We define the probability mass function of the discrete Gaussian distribution $\mathrm{D}_{\Lambda, \sigma, \mathrm{c}}$ :

$$
\forall x \in \Lambda, \mathrm{D}_{\Lambda, \sigma, c}(\mathrm{x})=\frac{\rho_{\sigma, c}(\mathrm{x})}{\rho_{\sigma, c}(\Lambda)} .
$$

Gentry etc.[5] proposed the Gaussian sampling algorithm that, given a basis of an arbitrary lattice, samples lattice points from a discrete Gaussian distribution.

\subsection{Learning with Errors}

For an integer $\mathrm{q}$, a positive integern and a distribution $\chi$ on $\mathbf{Z}_{q}$, learning with errors problem $\operatorname{LWE}_{q, \chi}$ aims to distinguish between the distribution $\mathrm{A}_{\mathrm{s}, \chi} \quad\left(s \in \mathbf{Z}_{q}^{n}\right.$ is some uniform secret value) and the uniform distribution on $\mathbf{Z}_{q}^{n} \times \mathbf{Z}_{q}$. The instance of LWE problem[7] contains access to an unspecified oracle $\mathrm{O}\left(\mathrm{O}_{s}\right.$ or $\left.{ }_{\$}\right) \mathrm{O}_{s}$ denotes a noisy pseudo-random sampler, it outputs samples $\left(u_{i}, v_{i}\right)=\left(u_{i}, u_{i}^{T} s+x_{i}\right) \in \mathbf{Z}_{q}^{n} \times \mathbf{Z}_{q}$, where $x_{i} \in \mathbf{Z}_{q}$ taken freshly from $\chi$ and $u_{i}$ is uniform in $\mathbf{Z}_{q}^{n} \cdot \mathrm{O}_{\$}$ represents a truly random sampler whose output is some truly uniform random samples from $\mathbf{Z}_{q}^{n} \times \mathbf{Z}_{q}$.

We say $A$ algorithm can decide the LWE problem, if $L W E-a d v[\mathrm{~A}]:=\left|\operatorname{Pr}\left[\mathrm{A}^{\mathrm{O}_{s}}=1\right]-\operatorname{Pr}\left[\mathrm{A}^{\mathrm{O}_{s}}=1\right]\right|$ is non-negligiblefor a random $s \in \mathbf{Z}_{q}^{n}$.

Regev[11] proved that for certain Gaussian noise distributions $\chi$ (denoted $\bar{\Psi}_{\alpha}$ ) on $\mathbf{Z}_{q}, \operatorname{LWE}_{q, \bar{\Psi}_{\alpha}}$ is as hard as using a quantum algorithm to solve the worst-case SIVP and GapSVP.

\subsection{Trapdoor Generation Algorithm}

In our construction, we use the TrapGen algorithm proposed by Alwen and Peikert[12] to generate a uniform matrix A, together with a good trapdoor basis T of $\Lambda_{q}^{\perp}(\mathrm{A})$.

Theorem 1.[12] Let odd $q \geq 3, m \geq\lceil 6 n \log q\rceil, \delta=1 / 3$. There is a probabilistic polynomial time algorithm TrapGen(q, n) outputs $\left(\mathrm{A} \in \mathbf{Z}_{q}^{n \times m}, T_{A} \in \mathbf{Z}^{m \times m}\right)$ such that $\mathrm{A}$ is statistically close to a uniform matrix, $T_{A}$ is a basis of $\Lambda_{q}^{\perp}(\mathrm{A})$ satisfying $\left\|\tilde{T}_{A}\right\|=O(\sqrt{n \log q}),\left\|T_{A}\right\| \leq O(n \log q)$.

\subsection{SampleLeft and SampleRight Algorithm}

Let matrixes $\mathrm{A}, B \in \mathbf{Z}_{q}^{n \times m}$ and $\mathrm{R}$ be a matrix in $\{-1,1\}^{m \times m}$, matrix $F=(\mathrm{A} \mid \mathrm{AR}+B) \in \mathbf{Z}_{q}^{n \times 2 m}$. In our construction, we have to sample short vectors in $\Lambda_{q}^{u}(\mathrm{~A})$ for $u \in \mathbf{Z}_{q}^{n}$ as the user's private key using SampleLeft algorithm or SampleRight algorithm for security game.

SampleLeft $\left(A, \mathrm{M}_{1}, T_{A}, \mathrm{u}, \sigma\right)[7]$ : Input a rank $n$ matrix $A \in \mathbf{Z}_{q}^{n \times m}, \mathrm{M}_{1} \in \mathbf{Z}_{q}^{n \times m_{1}}$, a short basis $T_{A}$ of $\Lambda_{q}^{\perp}(\mathrm{A})$, a Gaussian parameter $\sigma>\left\|\tilde{T}_{A}\right\| \cdot \omega\left(\sqrt{\log \left(m+m_{1}\right)}\right)$ and a vector $u \in \mathbf{Z}_{q}^{n}$, let $F_{1}=\left(\mathrm{A} \mid \mathrm{M}_{1}\right)$. This algorithm outputs a vector $e \in \mathbf{Z}^{m+m_{1}}$ satisfying $F_{1} \cdot e=u(\operatorname{modq})$, e is sampled from a distribution statistically close to $\mathrm{D}_{\Lambda_{q}^{u}\left(\mathrm{~F}_{1}\right), \sigma}$.

SampleRight $\left(A, B, R, T_{B}, u, \sigma\right)[7]$ : Input $A \in \mathbf{Z}_{q}^{n \times k}$ and a rank n matrix $B \in \mathbf{Z}_{q}^{n \times m}$, a matrix $R \in \mathbf{Z}^{k \times m}$ (Let $\left.s_{R}:=\|R\|=\sup _{\|x\|=1}\|R x\|\right), \quad T_{B} \quad$ is a basis of $\Lambda_{q}^{\perp}(\mathrm{B})$ and $u \in \mathbf{Z}_{q}^{n}$, the Gaussian parameter $\sigma>\left\|\tilde{T}_{B}\right\| \cdot s_{R} \omega(\sqrt{\log m})$. This algorithm outputs a vector $e \in \mathbf{Z}^{m+k}$, let F2be $(\mathrm{A} \mid \mathrm{AR}+B)$, e is sampled from a distribution statistically close to $\mathrm{D}_{\Lambda_{q}^{u}\left(\mathrm{~F}_{2}\right), \sigma}$.

\subsection{Lattice-based Hash function}

Definition [13] For security parameter $\lambda$, we choose a random matrix $M \in \mathbf{Z}_{q}^{\lambda \times m}, M_{i}$ denotes the $\mathrm{i}_{\text {th }}$ column ofM, for the input $x=\left(x_{1}, x_{2}, \ldots, x_{m}\right) \in \mathbf{Z}^{m}, h_{M}: \mathbf{Z}^{m} \rightarrow \mathbf{Z}_{q}^{\lambda}$ is defined as

$$
H_{M}(x)=\operatorname{M} x \bmod q=\sum_{i=1}^{m} x_{i} \mathrm{M}_{i} \bmod q .
$$


Let $\mu$ be polynomial in $\lambda$, denoted as $\mu=\operatorname{poly}(\lambda), \mathrm{q}$ be a $\lambda$-bits modulo, $m=2 \lambda^{2}$. When the input $x \in\{0,1, \ldots, \mu\}^{m}$, the LBH is collision-free and its security can be reduced to GAPSVP $14 \pi \sqrt{\lambda} \beta$.

\subsection{Full-rank Differences Function}

In our construction, we first use a collision resistant hash to convert the identity in $\{0,1\}^{*}$ to elements in $\mathbf{Z}_{q}^{n}$. Then use the full-rank difference function encoding the $i d=\left(\mathrm{d}_{1}, \mathrm{~d}_{2}, \cdots, \mathrm{d}_{n-1}\right) \in \mathbf{Z}_{q}^{n}$ to a matrix in $\mathbf{Z}_{q}^{n \times n}$. Let the polynomial $g_{d}(\mathrm{X})$ be $g_{d}(\mathrm{X})=\sum_{i=0}^{n-1} d_{i} x^{i} \in \mathbf{Z}_{q}[\mathrm{X}]$, $\operatorname{coeffs}\left(g_{d}\right)$ means the n-dimensional vector of coefficients of $g_{d}, f \in \mathbf{Z}_{q}[\mathrm{X}]$ be some polynomial of degree $\mathrm{n}$, the Full-Rank Differences function (FRD) $H($ id) as the definition in [7],

$$
H(\mathrm{id})=\left[\begin{array}{c}
\operatorname{coeffs}\left(\mathrm{g}_{d}\right) \\
\operatorname{coeffs}\left(\mathrm{X} \cdot \mathrm{g}_{d} \bmod f\right) \\
\operatorname{coeffs}\left(\mathrm{X}^{2} \cdot \mathrm{g}_{d} \bmod f\right) \\
\cdots \\
\operatorname{coeffs}\left(\mathrm{X}^{n-1} \cdot \mathrm{g}_{d} \bmod f\right)
\end{array}\right] \in \mathbf{Z}^{n \times n} .
$$

\section{The Main Construction}

\subsection{Description of Generic Distributed IBE}

The standard IBE includes four algorithms: Setup, Extract, Encrypt and Decrypt. Our construction introduces three new algorithms Distribution, Construct Dec and VerS.

The PKG runs the algorithm Setup to generate the master key, public key and all the public parameters.

The PKG runs the Extract algorithm after it receives the user's private key extraction request to generate the private key related with the user's identity.

In the application, the user can choose the number of decryption servers. Then the PKG or other authorized party that possesses the private key from PKG can run the Distribution algorithm to distribute the private key into $\mathrm{k}$ decryption servers $\Gamma_{i}$ for $1 \leq i \leq k \quad(k \leq 2 m$ in our distributed IBE). The $\mathrm{k}$ decryption servers use a broadcast channel in common, each secret share is kept secretly by the decryption server. In the Distribution algorithm, an improved secret sharing method will be applied to generate shares of the private key and verify the validity of shares.

Thei $_{\text {th }}$ server $\Gamma_{i}$ receives its secret share and runs the algorithm VerS to verify the validity of the secret share.

Any user who wants to send message to otherscan run the algorithm Encrypt after obtaining the public parameter and the receiver's identity. The legal receiver gets the ciphertext and sends a decryption request to the decryption servers. Then the decryption server $\Gamma_{i}$ implements the ConstructDec algorithm to generate corresponding decryption share and sends the decryption share to the user. The user can run the Decrypt algorithm combining the received valid decryption shares to recover the plaintext.

\subsection{Our Distributed IBE}

-Setup(n): On input $\mathrm{n}$ as a security parameter, set parameter, set $q, m, \sigma, \alpha$ (parameters set will be specified in the next section). Fist, this algorithm runs TrapGen(q,n) generating a uniformly random matrix $A_{0} \in \mathbf{Z}_{q}^{n \times m}$ altogether with a short basis $T_{A_{0}} \in \mathbf{Z}^{m \times m}$ for $\Lambda_{q}^{\perp}\left(\mathrm{A}_{0}\right) . T_{A_{0}}$ is the master key. Then it chooses two uniformly random matrixes $\mathrm{A}_{1}, B \in \mathbf{Z}_{q}^{n \times m}$ and a uniformly random vector $u \stackrel{R}{\leftarrow} \mathbf{Z}_{q}^{n}$. Finally, it returns the public parameters $\mathrm{PP}=\left(\mathrm{A}_{0}, \mathrm{~A}_{1}, \mathrm{~B}, \mathrm{u}\right)$ while keeping the master key $M K=\left(T_{A_{0}}\right)$ secretly.

-Extract(id, MK, PP): Given an identity $i d \in \mathbf{Z}_{q}^{n}$, a mater key $\mathrm{MK}$ and public parameters $\mathrm{PP}$, this algorithm uses SampleLeft $\left(\mathrm{A}_{0}, \mathrm{~A}_{1}+\mathrm{H}(\mathrm{id}) \mathrm{B}, \mathrm{T}_{A_{0}}, \mathrm{u}, \sigma\right)$ to sample a vector $e \in \mathbf{Z}^{2 m}$ as the private key $\mathrm{SK}_{\text {id }}$. $\mathrm{H}$ is a full-rank differences map. Let $\mathrm{F}_{i d}:=\left(\mathrm{A}_{0} \mid \mathrm{A}_{1}+\mathrm{H}(\mathrm{id}) \mathrm{B}\right)$, clearly the private key satisfies $\mathrm{F}_{i d} \cdot e=u(\operatorname{modq})$

-Distribution $\left(\mathrm{SK}_{\mathrm{id}}, \mathrm{k}, \mathrm{q}, 2 \mathrm{~m}\right)$ : Given a private key $\mathrm{SK}_{\mathrm{id}}$ and the number of decryption servers $\mathrm{k}$, this algorithm first uses function TrapGen by choosing $n^{\prime}, \mathrm{q}$ and $2 \mathrm{~m} \geq\left\lceil 6 n^{\prime} \log q\right\rceil$ as input to obtain a basis $\mathrm{T} \in \mathbb{Z}^{2 \mathrm{~m} \times 2 \mathrm{~m}}$. Then it selects arbitrary $\mathrm{k}$ linearly independent column-vectors of $\mathrm{T}$ forming a matrix $Q:=\left\{\mathrm{q}_{1}, \ldots, \mathrm{q}_{\mathrm{k}}\right\} \in \mathbb{Z}^{2 \mathrm{~m} \times \mathrm{k}}$ satisfying $\operatorname{Rank}(\mathrm{Q})=$ $\operatorname{Rank}\left(\left[\mathrm{Q} \mathrm{SK}_{\mathrm{id}}\right]\right)$. Then, it computes a vector $\mathrm{v}$ from $\mathrm{SK}_{\mathrm{id}}=\mathrm{Q} \cdot \mathrm{v}$ and selects $\mathrm{k}$ linearly independent vectors $\eta_{\mathrm{i}} \stackrel{\$}{\leftarrow}\{0,1\}^{\mathrm{k}}$ composing $\eta:=\left\{\eta_{1}, \ldots, \eta_{\mathrm{k}}\right\}$ (the Hamming weight of $\eta_{\mathrm{i}}$ satisfying $\left.1<w t\left(\eta_{\mathrm{i}}\right)<[\mathrm{k} / 2]\right)$.

It then computes each decryption server's secret share $\mathrm{d}_{\mathrm{i}}=\mathrm{Q} \cdot \eta_{\mathrm{i}} \in \mathbb{Z}^{2 \mathrm{~m}}$. Subsequently, it confidentially sends the secret share $d_{i}$ to decryption server $\Gamma_{i}$. By selecting a random matrix $\mathrm{M} \in \mathbb{Z}_{\mathrm{q}}^{\mathrm{k} \times 2 \mathrm{~m}}$ to pick a hash function $\mathrm{h}_{\mathrm{M}}: \mathbb{Z}^{2 \mathrm{~m}} \rightarrow \mathbb{Z}_{\mathrm{q}^{\prime}}^{\lambda}$, it computes $\mathrm{h}_{\mathrm{M}}\left(\mathrm{q}_{\mathrm{i}}\right) \quad(1 \leq \mathrm{i} \leq \mathrm{k})$ and $\mathrm{h}_{\mathrm{M}}\left(\mathrm{SK}_{\mathrm{id}}\right)$. The distributer makes common parameter $\mathrm{cp}=\left(\mathrm{v}, \eta, \mathrm{h}_{\mathrm{M}}, \mathrm{h}_{\mathrm{M}}\left(\mathrm{q}_{\mathrm{i}}\right), \mathrm{h}_{\mathrm{M}}\left(\mathrm{SK}_{\mathrm{id}}\right)\right)$ public and keeps $\mathrm{Q}$ and $\mathrm{SK}_{\text {id }}$ secretly.

-VerS $\left(h_{M}\left(d_{i}\right), \eta, h_{M}\left(q_{j}\right)\right)$ : This algorithm computes $h_{M}\left(d_{i}\right)$ and checks if $h_{M}\left(d_{i}\right)=\sum_{j=1}^{k} \eta_{i j} \cdot h_{M}\left(q_{j}\right)$, then accepts the secret share $d_{i}$ and returns an acknowledgement, otherwise outputs "Invalid shares".

-Encrypt(PP, id, b): On input a message $b \in\{0,1\}$, an identity id, public parameters PP, this algorithm picks a uniformly random $\mathrm{s} \stackrel{\mathrm{R}}{\leftarrow} \mathbb{Z}_{\mathrm{q}}^{\mathrm{n}}$, a uniformly random matrix 
$\mathrm{R} \stackrel{\mathrm{R}}{\leftarrow}\{-1,1\}^{\mathrm{m} \times \mathrm{m}}$ and noise vectors $\mathrm{x} \stackrel{\bar{\Psi}_{\alpha}}{\leftarrow} \mathbb{Z}_{\mathrm{q}}, \mathrm{y} \stackrel{\bar{\Psi}_{\alpha}^{\mathrm{m}}}{\longleftarrow} \mathbb{Z}_{\mathrm{q}}^{\mathrm{m}}$, let $\mathrm{z}=\mathrm{R}^{\mathrm{T}} \mathrm{y}$, and subsequently computes $\mathrm{c}_{0} \leftarrow \mathrm{u}^{\mathrm{T}} \mathrm{s}+\mathrm{x}+$ $\mathrm{b}\lfloor\mathrm{q} / 2] \in \mathbb{Z}_{\mathrm{q}}, \quad \mathrm{c}_{1} \leftarrow \mathrm{F}_{\mathrm{id}}^{\mathrm{T}} \mathrm{s}+\left[\begin{array}{l}\mathrm{y} \\ \mathrm{z}\end{array}\right] \in \mathbb{Z}_{\mathrm{q}}^{2 \mathrm{~m}}$ and returns a ciphertext $\mathrm{C}=\left(\mathrm{c}_{0}, \mathrm{c}_{1}\right)$.

-ConstructDec $\left(\eta, v, c_{1}, d_{j}\right)$ : On input $\eta, v, c_{1}$, this algorithm computes $E=\left[\eta_{1}, \ldots, \eta_{k}\right] \cdot A_{2}{ }^{T}(E$ denotes $k \times k$ unit matrix) to obtain the matrix $A_{2}=\left[a_{1}, \ldots, a_{k}\right]$. The decryption server $\Gamma_{j}$ computes decryption share $W_{j}$, $\mathrm{W}_{\mathrm{j}}=\left[\left(\sum_{\mathrm{i}=1}^{\mathrm{k}} \mathrm{v}_{\mathrm{i}} \cdot \mathrm{a}_{\mathrm{j}}^{(\mathrm{i})}\right) \cdot \mathrm{d}_{\mathrm{j}}^{(1)}, \ldots,\left(\sum_{\mathrm{i}=1}^{\mathrm{k}} \mathrm{v}_{\mathrm{i}} \cdot \mathrm{a}_{\mathrm{j}}^{(\mathrm{i})}\right) \cdot \mathrm{d}_{\mathrm{j}}^{(2 \mathrm{~m})}\right]$. Then it computes $\mathrm{h}_{\mathrm{M}}\left(\mathrm{W}_{\mathrm{j}}^{\mathrm{T}}\right)$ and outputs $\zeta_{\mathrm{j}, \mathrm{c}_{1}}, \zeta_{\mathrm{j}, \mathrm{c}_{1}}=\left(\mathrm{W}_{\mathrm{j}}\right.$. $\left.c_{1}, h_{M}\left(W_{j}^{T}\right)\right)$.

$-\operatorname{Decrypt}\left(\zeta_{\mathrm{j}, \mathrm{c}_{1}}, \mathrm{PP}, \mathrm{C}\right)$ : Given $\zeta_{\mathrm{j}, \mathrm{c}_{1}}, \mathrm{PP}, \mathrm{C}=\left(\mathrm{c}_{0}, \mathrm{c}_{1}\right)$, it computes $\sum_{j=1}^{k} h_{M}\left(W_{j}^{T}\right)$ and checks if $\sum_{j=1}^{k} h_{M}\left(W_{j}^{T}\right)=$ $\mathrm{h}_{\mathrm{M}}\left(\mathrm{SK}_{\mathrm{id}}\right)$. If the test holds, accept the decryption shares and do as follows: compute $\omega \leftarrow \mathrm{c}_{0}-\sum_{\mathrm{j}=1}^{\mathrm{k}}\left(\mathrm{W}_{\mathrm{j}} \cdot \mathrm{c}_{1}\right) \in \mathbb{Z}_{\mathrm{q}}$ and compare $\omega$ and $\lfloor q / 2\rfloor$, if $|\omega-\lfloor q / 2\rfloor|<\lfloor q / 4\rfloor$, output 1 , otherwise output 0 .

\subsection{Correctness}

Due to $\sum_{\mathrm{j}=1}^{\mathrm{k}}\left(\mathrm{W}_{\mathrm{j}} \cdot \mathrm{c}_{1}\right)=\left(\sum_{\mathrm{j}=1}^{\mathrm{k}} \mathrm{W}_{\mathrm{j}}\right) \cdot \mathrm{c}_{1}$, we can obtain $\sum_{\mathrm{j}=1}^{\mathrm{k}} \mathrm{W}_{\mathrm{j}}=(\mathrm{Q} \cdot \mathrm{v})^{\mathrm{T}}$ from the deduction for $\sum_{\mathrm{j}=1}^{\mathrm{k}} \mathrm{W}_{\mathrm{j}}$, as follows:

$$
\begin{aligned}
\sum_{j=1}^{k} W_{j} & =\left[\begin{array}{c}
\left(\sum_{i=1}^{k} v_{i} \cdot \mathrm{a}_{1}^{(\mathrm{i})}\right) \cdot \mathrm{d}_{1}^{(1)}+\left(\sum_{i=1}^{k} v_{i} \cdot \mathrm{a}_{2}^{(\mathrm{i})}\right) \cdot \mathrm{d}_{2}^{(1)}+\cdots+\left(\sum_{i=1}^{k} v_{i} \cdot \mathrm{a}_{k}^{(i)}\right) \cdot \mathrm{d}_{k}^{(1)} \\
\left(\sum_{i=1}^{k} v_{i} \cdot \mathrm{a}_{1}^{(\mathrm{i})}\right) \cdot \mathrm{d}_{1}^{(2)}+\left(\sum_{i=1}^{k} v_{i} \cdot \mathrm{a}_{2}^{(\mathrm{i})}\right) \cdot \mathrm{d}_{2}^{(2)}+\cdots+\left(\sum_{i=1}^{k} v_{i} \cdot \mathrm{a}_{k}^{(\mathrm{i})}\right) \cdot \mathrm{d}_{k}^{(2)} \\
\vdots \\
\left(\sum_{i=1}^{k} v_{i} \cdot \mathrm{a}_{1}^{(i)}\right) \cdot \mathrm{d}_{1}^{(2 \mathrm{~m})}+\left(\sum_{i=1}^{k} v_{i} \cdot \mathrm{a}_{2}^{(\mathrm{i})}\right) \cdot \mathrm{d}_{2}^{(2 \mathrm{~m})}+\cdots+\left(\sum_{i=1}^{k} v_{i} \cdot \mathrm{a}_{k}^{(\mathrm{i})}\right) \cdot \mathrm{d}_{k}^{(2 \mathrm{~m})}
\end{array}\right]^{T} \\
= & {\left[\left(\sum_{i=1}^{k} v_{i} \cdot \mathrm{a}_{1}^{(\mathrm{i})}\right),\left(\sum_{i=1}^{k} v_{i} \cdot \mathrm{a}_{2}^{(\mathrm{i})}\right), \cdots,\left(\sum_{i=1}^{k} v_{i} \cdot \mathrm{a}_{k}^{(\mathrm{i})}\right)\right] \cdot\left[d_{1}, d_{2}, \cdots, d_{k}\right]^{T} } \\
= & v^{T} \cdot\left[\mathrm{a}_{1}, \mathrm{a}_{2}, \cdots, \mathrm{a}_{k}\right] \cdot\left[d_{1}, d_{2}, \cdots, d_{k}\right]^{T} \\
= & \left(\left[d_{1}, d_{2}, \cdots, d_{k}\right] \cdot\left[\mathrm{a}_{1}, \mathrm{a}_{2}, \cdots, \mathrm{a}_{k}\right]^{T} \cdot v\right)^{T} \\
= & \left(\left[\mathrm{q}_{1}, \mathrm{q}_{2}, \cdots, \mathrm{q}_{k}\right] \cdot\left[\eta_{1}, \eta_{2}, \cdots, \eta_{k}\right] \cdot\left[\mathrm{a}_{1}, \mathrm{a}_{2}, \cdots, \mathrm{a}_{k}\right]^{T} \cdot v\right)^{T} \\
= & \left(\left[\mathrm{q}_{1}, \mathrm{q}_{2}, \cdots, \mathrm{q}_{k}\right] \cdot \mathrm{E} \cdot v\right)^{T} \\
= & (\mathrm{Q} \cdot v)^{T} .
\end{aligned}
$$

When $\sum_{\mathrm{j}=1}^{\mathrm{k}} \mathrm{W}_{\mathrm{j}}=(\mathrm{Q} \cdot \mathrm{v})^{\mathrm{T}}=\left(\mathrm{SK}_{\mathrm{id}}\right)^{\mathrm{T}}$ is easily gotten,we have $\omega$ as follows:

$$
\begin{aligned}
& \omega=c_{0}-\left(S K_{i d}\right)^{T} c_{1} \\
& =u^{T} s+x+b\lfloor q / 2\rfloor-S K_{i d}^{T}\left(F_{i d}^{T} s+\left[\begin{array}{l}
y \\
z
\end{array}\right]\right) \\
& =b\lfloor q / 2\rfloor+x-S K_{i d}^{T}\left[\begin{array}{c}
y \\
z
\end{array}\right] .
\end{aligned}
$$

Setting $\left(x-e_{i d}^{T}\left[\begin{array}{l}\mathrm{y} \\ z\end{array}\right]\right)$ as the error term, the decryption operates successfully when the error term is less than q/
5 with high probability. By Lemma 22 in [8], the norm of error term is bounded by $\mathrm{q} \sigma \mathrm{m} \alpha \omega\left(\sqrt{\operatorname{logm}}+\mathrm{O}\left(\sigma \mathrm{m}^{3 / 2}\right)\right]$.

\subsection{Parameters}

To make the system work correctly, following conditions have to be satisfied:

1) Let $m>6 n \log q$, so the function TrapGen can operate.

2) Set the Gaussian parameter $\sigma>m \omega(\sqrt{\operatorname{logm}})$, so SampleLeft and SampleRight can operate.

3) Let $q>2 \sqrt{n} / \alpha$, so Regev's reduction holds.

4) Set $\alpha<[\sigma m \omega(\sqrt{\operatorname{logm}})]^{-1}$ and $q=\Omega\left(\sigma m^{\frac{3}{2}}\right)$ for the error term is less than $q / 5$.

5) Let $\mu=O($ nlogq $)$ and $\mu>m \omega(\sqrt{\operatorname{logm}}), \lambda^{2}=m$ to satisfy that the input for lattice-based hash function is collision-free.

We assume that $n^{\delta}>\lceil\log q\rceil=0(\log n), n$ is the security parameter, we set the parameters $(m, q, \sigma, \alpha, \mu, \lambda)$ as follows:

$$
\begin{aligned}
& m=6 n^{1+\delta}, q=m^{2.5} \cdot \omega(\sqrt{\log n}), \sigma=m \cdot \omega(\sqrt{\log n}) \\
& \alpha=\left[m^{2} \cdot \omega(\sqrt{\log n})\right]^{-1}, \mu=m 0(\log n), \lambda=\sqrt{m} .
\end{aligned}
$$

We set $\lambda$ and $m$ to the nearest larger integer, $q$ to the nearest larger prime.

\section{Security Reduction}

We define the security notion for our distributed IBE. INDr-sIDD-CPA is the notion of indistinguish ability of ciphertext under a selective identity chosen plaintext attack for the distributed IBE. INDr denotes indistinguishable from random, that means the challenge ciphertext is indistinguishable from random elements in the ciphertext space.

Definition. Let $\mathcal{A}$ be an INDr-sIDD-CPA PPT adversary. Consider the following Game 0 in which the adversary $\mathcal{A}$ interacts with the Chanllenger.

Initial: The adversary $\mathcal{A}$ outputs a target identity $\mathrm{id}^{*}$.

Setup: Then Challenger runs algorithm $\operatorname{Setup(n),~} \mathrm{n}$ is a security parameter, and sends the resulting public system parameters $\mathrm{PP}=\left(\mathrm{A}_{0}, \mathrm{~A}_{1}, \mathrm{~B}, \mathrm{u}\right)$ to $\mathcal{A}$. However, it keeps the master key $\mathrm{MK}$ secretly.

Phase 1: $\mathcal{A}$ corrupts $\mathrm{k}-1$ decryption servers.

Phase 2: The Challenger runs the Extract algorithm to obtain a private key $\mathrm{d}_{\mathrm{id}^{*}}$ corresponding to the target identity $\mathrm{id}^{*}$. Subsequently, the Challenger runs the Distribution algorithm on $\mathrm{d}_{\mathrm{id}^{*}}$ with the input parameter $\mathrm{k}$. It then obtains a set of secret shares $\left\{\mathrm{d}_{\mathrm{i}} \mid 1 \leq \mathrm{i} \leq \mathrm{k}\right\}$ and sends the $\mathrm{k}-1$ secret shares of corrupted decryption servers with the corresponding $\mathrm{h}_{\mathrm{M}}\left(\mathrm{d}_{\mathrm{i}}\right)$ to $\mathcal{A}$. Besides, the secret share of the uncorrupted server is kept secretly.

Phase 3: $\mathcal{A}$ issues adaptive private key extraction queries $\mathrm{q}_{1}, \ldots, \mathrm{q}_{\mathrm{m}}$, where $\mathrm{q}_{\mathrm{i}}$ denotes the $\mathrm{i}^{\text {th }}$ query on 
$\mathrm{id}_{\mathrm{i}}\left(\mathrm{id}_{\mathrm{i}} \neq \mathrm{id}^{*}\right)$. Then the Challenger runs Extract algorithm on $\mathrm{id}_{\mathrm{i}}$ to obtain a corresponding private key $\mathrm{e}_{\mathrm{i}}$ to respond the query $\mathrm{q}_{\mathrm{i}}$. It sends $\mathrm{e}_{\mathrm{i}}$ to $\mathcal{A}$.

Challenge: After $\mathcal{A}$ finishes the Phase 3, it outputs a plaintext $\mathcal{M}$ to be challenged. The Challenger chooses a random bit $r \in\{0,1\}$ and a random ciphertext $\mathcal{C} \in \mathbb{Z}_{\mathrm{q}} \times$ $\mathbb{Z}_{\mathrm{q}}^{2 \mathrm{~m}}$. If $\mathrm{r}=0$ it gives the challenge ciphertext $\mathcal{C}^{*}=$ $\operatorname{Encrypt}\left(\mathrm{PP}, \mathrm{id}^{*}, \mathcal{M}\right)$ to $\mathcal{A}$. If $\mathrm{r}=1$, it sends the random ciphertext $\mathcal{C}^{*}=\mathcal{C}$ to $\mathcal{A}$.

Phase 4: $\mathcal{A}$ issues arbitrary adaptive private key extraction queries $\mathrm{q}_{\mathrm{m}+1}, \ldots, \mathrm{q}_{\mathrm{n}}$, where $\mathrm{q}_{\mathrm{i}}$ denotes the $\mathrm{i}^{\text {th }}$ query on $\mathrm{id}_{\mathrm{i}}\left(\mathrm{id}_{\mathrm{i}} \neq \mathrm{id}^{*}\right)$. The Challenger responds as in Phase 3.

Guess: $\mathcal{A}$ outputs a guess $\mathrm{r}^{\prime} \in\{0,1\}$. If $\mathrm{r}=\mathrm{r}^{\prime}$, it wins the game.

We define the advantage of an INDr-sIDD-CPA adversary $\mathcal{A}$ in attacking the distributed IBE $\mathcal{E}$ as $\operatorname{Adv}_{\mathcal{E}, \mathcal{A}}(n)=\left|\operatorname{Pr}\left[\mathrm{r}=\mathrm{r}^{\prime}\right]-1 / 2\right|$.

Theorem 2. In the standard model, our distributed IBE with parameters setting as in Sectionparameters is INDr-sIDD-CPA secure if the $\left(\mathbb{Z}_{\mathrm{q}}, \mathrm{n}, \bar{\Psi}_{\alpha}\right)-\mathrm{LWE}$ assumption holds.

Proof.

Game 0. It is the original INDr-sIDD-CPA game as previously mentioned.

Game 1. In Game 1, we change slightly the way to generate $A_{1}$ which is one of public parameters in PP. Let the Challenger in Game 1 select a random matrix $R^{*} \in$ $\{-1,1\}^{\mathrm{m} \times \mathrm{m}}$ at the Setup phase, $\mathrm{id}^{*}$ is the target identity that $\mathcal{A}$ wants to attack. $\mathrm{A}_{1}$ is constructed as $\mathrm{A}_{1}=\mathrm{A}_{0} \mathrm{R}^{*}-$ $\mathrm{H}\left(\mathrm{id}^{*}\right) \mathrm{B}$. The Challenger use SampleRight algorithm to generate $e_{i d}$ to respond to a private key extraction query. The rest of the game is the same as Game 0 .

By Lemma 13 in [7], Game 1 and Game 0 is statistically indistinguishable.

Game 2. We change the way to generate $A_{0}$ and $B$ in PP. The Challenger chooses a random matrix in $\mathbb{Z}_{\mathrm{q}}^{\mathrm{n} \times \mathrm{m}}$ as $\mathrm{A}_{0}$ and runs the TrapGen(q,n) to generate a uniformly random matrix $B \in \mathbb{Z}_{q}^{n \times m}$ altogether with a trapdoor $T_{B}$. The construction of $A_{1}$ is the same as Game 1.

The Challenger runs SampleRight $\left(\mathrm{A}_{0},(\mathrm{H}(\mathrm{id})-\right.$ $\left.\mathrm{H}\left(\mathrm{id}^{*}\right) \mathrm{B}, \mathrm{R}^{*}, \mathrm{~T}_{\mathrm{B}}, \mathrm{u}, \sigma\right)$ to sample a vector $\mathrm{e} \in \mathbb{Z}_{\mathrm{q}}^{2 \mathrm{~m}}$ and sends $\mathrm{SK}_{\mathrm{id}}=\mathrm{e}$ to $\mathcal{A}$ to respond to the private key query.

Let $\mathrm{F}_{\mathrm{id}}=\left(\mathrm{A}_{0} \mid \mathrm{A}_{0} \mathrm{R}^{*}+\left(\mathrm{H}(\mathrm{id})-\mathrm{H}\left(\mathrm{id}^{*}\right)\right) \cdot \mathrm{B}\right)$, according to Theorem 19 in [7], when $\sigma>\left\|\widetilde{T}_{B}\right\| s_{R} \omega(\sqrt{\operatorname{logm}})$, the generated e is distributed statistically close to $\mathrm{D}_{\wedge_{\mathrm{q}}^{u}\left(\mathrm{~F}_{\mathrm{id}}\right), \sigma}$, as in Game 1.

So Game 2 and Game 1 is statistically indistinguishable.

Game 3. In Game 3, the challenge ciphertext $\mathcal{C}^{*}=$ $\left(\mathrm{c}_{0}^{*}, \mathrm{c}_{1}^{*}\right)$ is selected by random independent elements in $\mathbb{Z}_{\mathrm{q}} \times \mathbb{Z}_{\mathrm{q}}^{2 \mathrm{~m}}$. The rest of Game 3 is the same as Game 2. The advantage of $\mathcal{A}$ in this game is zero.

We can refer to the reduction from the LWE problem in [7] to show Game 2 and Game 3 are indistinguishable for $\mathcal{A}$. The reduction from the LWE problem shows as follows:
We assume that $\mathcal{A}$ 's advantage in distinguishing Game 2 and Game 3 is non-negligible and construct an LWE algorithm $\mathcal{B}$ using $\mathcal{A}$. $\mathcal{B}$ receives LWE samples $\left(\mathrm{u}_{\mathrm{i}}, \mathrm{v}_{\mathrm{i}}\right) \in \mathbb{Z}_{\mathrm{q}}^{\mathrm{n}} \times \mathbb{Z}_{\mathrm{q}}, \mathrm{i}=0, \ldots, \mathrm{m}$ from an oracle $\mathcal{O}$ as mentioned in Section 2.4.

Initial: The adversary $\mathcal{A}$ outputs a target identity $\mathrm{id}^{*}$ to $\mathcal{B}$.

Setup: $\mathcal{B}$ constructs public parameters $\mathrm{PP}=\left(\mathrm{A}_{0}, \mathrm{~A}_{1}, \mathrm{~B}, \mathrm{u}_{0}, \mathrm{R}^{*}\right)$, where $\mathrm{A}_{0}=\left[\mathrm{u}_{1}, \mathrm{u}_{2}, \cdots, \mathrm{u}_{\mathrm{m}}\right] \in$ $\mathbb{Z}_{\mathrm{q}}^{\mathrm{n} \times \mathrm{m}}$, the construction of $\mathrm{A}_{1}$ andB is the same as in Game 2, $\mathrm{u}_{0}$ is the $0^{\text {th }}$ LWE sample, $\mathrm{R}^{*} \in\{-1,1\}^{\mathrm{m} \times \mathrm{m}}$.

Queries: $\mathcal{B}$ responds to the private key extraction queries as in Game 2.

Challenge: $\mathcal{A}$ outputs a plaintext $\mathrm{b}^{*} \in\{0,1\}$, then $\mathcal{B}$ sends a challenge ciphertext $\mathcal{C}^{*}=\left(\mathrm{c}_{0}^{*}, \mathrm{c}_{1}^{*}\right)$ for the target id ${ }^{*}$ to $\mathcal{A}$. We set $\mathrm{v}^{*}=\left[\begin{array}{c}\mathrm{v}_{1} \\ \cdot \\ \cdot \\ \mathrm{v}_{\mathrm{m}}\end{array}\right] \in \mathbb{Z}_{\mathrm{q}}^{\mathrm{m}}, \mathrm{c}_{0}^{*}=\mathrm{v}_{0}+\mathrm{b}^{*}[\mathrm{q} / 2] \in$ $\mathbb{Z}_{\mathrm{q}}$ and $\mathrm{c}_{1}^{*}=\left[\begin{array}{c}\mathrm{v}^{*} \\ \left(\mathrm{R}^{*}\right)^{\mathrm{T}} \mathrm{v}^{*}\end{array}\right] \in \mathbb{Z}_{\mathrm{q}}^{2 \mathrm{~m}}$.

When the LWE oracle $\mathcal{O}=\mathcal{O}_{\mathrm{s}}$ (which is a pseudorandom sampler), then $\mathcal{C}^{*}$ is distributed the same as the challenge ciphertext in Game 2. When $\mathcal{O}=\mathcal{O}_{\$}$, the challenge ciphertext is the same as in Game 3.

Guess: $\mathcal{A}$ guesses if it interacts with a Game 2 Challenger or Game 3 Challenger after issuing arbitrary adaptive private key extraction queries $\mathrm{q}_{\mathrm{m}+1}, \ldots, \mathrm{q}_{\mathrm{n}}$. then $\mathcal{B}$ outputs the guess of $\mathcal{A}$ to answer the LWE challenge.

The advantage of $\mathcal{B}$ to solve LWE problem is the same as the advantage of $\mathcal{A}$ to distinguish Game 2 and Game 3.

\section{Efficiency Analysis}

We show the comparison of our lattice-based distributed IBE and Baek and Zheng Yulliang's pairing-based distributed IBE in calculation cost.

Table1:Comparison of Our Scheme And Bz Scheme comparison

\begin{tabular}{|c|c|c|}
\hline & Baek's scheme & Our scheme \\
\cline { 2 - 3 } & Calculation cost & Calculation cost \\
\hline $\begin{array}{c}\text { Private key } \\
\text { distribution }\end{array}$ & $\mathrm{kM}_{1}$ & $(\mathrm{k}+1) \mathrm{Mul}$ \\
\hline $\begin{array}{c}\text { Secret share } \\
\text { generation }\end{array}$ & $\mathrm{tP}$ & $\begin{array}{c}\text { Mul }+2 \mathrm{~m}(\mathrm{kM} \\
+(\mathrm{k}-1) \mathrm{A})\end{array}$ \\
\hline $\begin{array}{c}\text { Verification of } \\
\text { secret share }\end{array}$ & $\begin{array}{c}(\mathrm{n}+4 \mathrm{t}+4) \mathrm{P}+(\mathrm{t} \\
+2) \mathrm{H}\end{array}$ & $(2 \mathrm{k}+1) \mathrm{H}$ \\
\hline Combination & $\mathrm{tL}+\mathrm{tM}_{1}+(\mathrm{t}$ & $\mathrm{kMul}$ \\
& $-1) \mathrm{M}_{\mathrm{T}}$ & \\
\hline
\end{tabular}

Table2:Notation Illustration

\begin{tabular}{|c|l|c|c|}
\hline Notation & Operation & Notation & Operation \\
\hline $\mathrm{L}$ & $\begin{array}{c}\text { Lagrange } \\
\text { interpolation } \\
\text { operation }\end{array}$ & $\mathrm{H}$ & $\begin{array}{c}\text { Hash } \\
\text { operation }\end{array}$ \\
\hline $\mathrm{P}$ & pairing & Mul & matrix \\
\hline
\end{tabular}




\begin{tabular}{|c|l|c|c|}
\hline $\mathrm{M}_{1}$ & operation & & multiplication \\
\hline $\begin{array}{c}\text { the point } \\
\text { multiplication in } \\
\mathrm{G}_{1}\end{array}$ & $\mathrm{M}$ & $\begin{array}{c}\text { multiplica } \\
\text { tion operation } \\
\text { in } \mathbb{Z}\end{array}$ \\
\hline $\mathrm{M}_{\mathrm{T}}$ & $\begin{array}{c}\text { multiplicati } \\
\text { on in } \mathrm{G}_{\mathrm{T}}\end{array}$ & $\mathrm{A}$ & $\begin{array}{c}\text { addition } \\
\text { operation in } \mathbb{Z}\end{array}$ \\
\hline
\end{tabular}

The $k$ represents the number of decryption servers, $t$ represents the threshold.

Table II explains the meaning of the notations in Table I. For example, it shows that at private key distribution phase, Baek's scheme costs $\mathrm{kM}_{1}$ ( $\mathrm{k}$ times point multiplication in $\left.\mathrm{G}_{1}\right)$ and it costs $(\mathrm{k}+1)$ times matrix multiplication. The advantages in our scheme appears in secret share generation and verification of secret share phase. As we know, the pairing operation is much more complicate than simple matrix multiplication and addition. Our distributed IBE avoids the complex pairing operation, so our scheme reduces the calculation cost and improves the efficiency.

\section{Conclusion}

In this paper, we improve the lattice-based secret sharing method and propose a lattice-based distributed IBE provably secure against selective identity chosen-plaintext attack. Through theoretical analysis, compared with the Baek and Zheng Yuliang's scheme, our scheme reduces calculation cost.

\section{Acknowledgement}

I would like to express my gratitude to all those who helped me for finishing this paper, especially to Professor Zhang Quan, my supervisor, for his encouragement and guidance.

\section{References}

[1] Shamir A. Identity-based cryptosystems and signature schemes[C]//Advances in cryptology. Springer Berlin Heidelberg, 1984: 47-53.

[2] Boneh D, Franklin M. Identity-based encryption from the Weil pairing[C]//Advances inCryptology-CRYPTO 2001. Springer Berlin Heidelberg, 2001: 213-229.

[3] Shamir A. How to share a secret[J]. Communications of the ACM, 1979, 22(11): 612-613.

[4] Baek J, Zheng Y. Identity-based threshold decryption[M]//Public Key Cryptography-PKC 2004. Springer Berlin Heidelberg, 2004: 262-276.

[5] Gentry C, Peikert C, Vaikuntanathan V. Trapdoors for hard lattices and new cryptographic constructions[C]//Proceedings of the fortieth annual ACM symposium on Theory of computing. ACM, 2008: 197-206.

[6] Cash D, Hofheinz D, Kiltz E, et al. Bonsai trees, or how to delegate a lattice basis[J]. Journal of cryptology, 2012, 25(4): 601-639.

[7] Agrawal S, Boneh D, Boyen X. Efficient lattice (H) IBE in the standard model[M]//Advances in Cryptology-EUROCRYPT 2010. Springer Berlin Heidelberg, 2010: 553-572.

[8] El Bansarkhani R, Meziani M. An efficient lattice-based secret sharing construction[M]//nformation Security Theory and Practice. Security, Privacy and Trust in Computing Systems and Ambient Intelligent Ecosystems. Springer Berlin Heidelberg, 2012: 160-168.

[9] Blakely G R. Safeguarding cryptographic keys[C]//Proc. AFIPS. 1979, 48: 313-317.

[10] Asmuth C, Bloom J. A modular approach to key safeguarding $[\mathrm{J}]$. IEEE transactions on information theory, 1983, 30(2): 208-210.

[11] Regev O. On lattices, learning with errors, random linear codes, and cryptography[J]. Journal of the ACM (JACM), 2009, 56(6): 34.

[12] Alwen J, Peikert C. Generating shorter bases for hard random lattices $[\mathrm{J}]$. Theory of Computing Systems, 2011, 48(3): 535-553.

[13] Zhou F. Lattice theory and crypotography [M]. Science Press, 2013 